\title{
Numerical analysis of passenger kinematics and injury risks during a railway vehicle collision: The effect of safety belts
}

\author{
J. Vychytil ${ }^{a, *}$, S. Špirk ${ }^{a}$ \\ ${ }^{a}$ Regional Technological Institute, University of West Bohemia, Univerzitní 8, 30100 Plzeň, Czech Republic
}

Received 2 September 2019; accepted 23 April 2020

\begin{abstract}
The aim of this paper is to contribute to investigation of passive safety of passengers in railway vehicles using numerical simulations. At first, a typical interior of a railway vehicle is created in the Virtual Performance Solution software. It includes finite element models of seats. Their mechanical properties have been determined experimentally. Particular attention is paid to the table in the backseat as a potentially harmful element. In numerical simulations, both folded and unfolded states of the table are considered. The passenger is represented with the virtual human body model Virthuman. Its structure is composed of rigid bodies and deformable segments to account for deformability of soft tissues. It allows for the prediction of injury risk. The numerical simulations aim to investigate the potential benefit of standard seat belts in a railway vehicle. Therefore, the passenger is seated considering three configurations: seating with no constraints, belted with a two-point belt and a three-point belt. The interior is loaded with a crash-pulse prescribed by the GM/RT2100 regulation. The results are discussed in terms of passenger kinematics and injury risk.
\end{abstract}

(C) 2020 University of West Bohemia. All rights reserved.

Keywords: railway vehicle safety, open coach, impact, Virthuman

\section{Introduction}

Passive safety of railway vehicle interiors is ensured by national regulations. In the Czech Republic, for instance, the ČSN EN 15227 regulation ensures safety of railway vehicle structures [6]. It prescribes several physical tests considering various scenarios and impact velocities (frontal impact of two coaches, impact with a heavy obstacle such as lorry etc.). Impact scenarios may be represented with acceleration pulses [12]. The regulation then requires satisfying particular criteria to ensure passenger's safety such as energy absorption in the vehicle structure, limit value of deceleration, preserving of a space for survival etc. The regulation is hence focused on the strength of structures within an interior. The effect of the impact scenario on the human body in terms of its kinematics and injury risk is not investigated. The impact test may be done via numerical simulation, however, the agreement with experiment has to be documented. In the UK, on the other hand, the GM/RT2100 regulation prescribes the impact test with passengers represented with Hybrid III dummies [14]. Namely, 50-th and 95-th percentile males are seated. After the loading with prescribed pulse the injury criteria are evaluated. To fulfill the requirements of the regulation, injury criteria cannot exceed certain limits. For instance, it has to be HIC $15<500$. The impact test has to be conducted experimentally. The similar approach is used also in automotive industry in the framework of regulation tests and consumer testing such as EuroNCAP [9]. A vehicle undergoes several impact scenarios and the injury criteria

\footnotetext{
*Corresponding author. Tel.: +420 377634 838, e-mail: jvychyti@ntc.zcu.cz. https://doi.org/10.24132/acm.2020.562
} 
are evaluated using dummies to represent occupants. The vehicle is rated accordingly to the obtained values of injury criteria.

Physical tests are crucial for the assesment of railway vehicles safety. However, their disadvantage is their high cost in terms of both time and finances. Therefore, numerical simulations are becoming more important as a support of these tests. Significant asset of numerical simulations is the so-called virtual prototyping. That is, the numerical simulations can be used to test a prototype of a safety element prior to its manufacturing. Also, they can be used to simulate complex collision scenarios that cannot be reproduced experimentally. In [13], for instance, impact scenario of a commuter train and a lorry is simulated considering up to 10 passengers. Injury risk of passengers caused by the collision with a table in a railway vehicle interior is investigated using numerical simulations in [15]. The example of virtual prototyping can be found in [16]. Here, the authors use numerical simulations in the development process of the seat for railway vehicles.

In automotive industry, safety belts are with no doubts established and inherent part of passive safety elements. They keep the occupant at his or her seated position during the impact scenario. That is, they prevent excessive motion of an occupant and its impact with the parts of vehicle interior. However, application of such safety elements in railway vehicles is questionable. There is an important aspect of the passenger comfort in railway vehicles which might be affected. Also, application of safety belts represent additional cost for manufacturer. Hence, the crucial question is related to the potential benefit of safety belts. Would their application increase passenger safety in a collision scenario?

The study presented in this paper aims to answer that question. In fact, it is an opportunity for the application of numerical methods. Using simulations, we are able to test different safety belts in a collision scenario and analyze their benefit in terms of passenger's injury risk. To review the current state of art, let us focus on long-distance buses. Two-point belts are common parts of their interior in the last years. Moreover, arrangement of a long-distance bus interior resembles the open coach type interior in railway vehicles. Therefore, findings regarding application of safety belts in long-distance buses might be useful also for their consideration in railway vehicle interiors.

The safety of buses is a focus of the ECE R66 regulation [8]. It prescribes the rollover test scenario. The so-called anthropometric balast is belted with a two-point belt to represent passenger. The rollover accident scenario is also an object of the study [2]. The authors analyzed bus accidents for 10 years. In their conclusion the application of two-point belts decreases the number of MAIS 2+ injury in a rollover accident scenario. The effect of safety belts on an injury sustained by passengers in buses is investigated also in [1]. According to this study, two-point belts are relatively cheap and easy to mount. They decrease the injury risk in the rollover accident scenario since they prevent motion of the passenger within the interior. On the other hand, they can contribute to the head and chest injuries during the frontal crash due to the impact of passenger and the backseat. Therefore, three-point belt is more suitable. Injury risk of a passenger during a frontal crash of a bus is also an object of study in [7]. The authors identify the impact of the head with the backseat as a potential risk and hence propose design changes. Similar results regarding frontal crash scenario might be expected also in numerical simulations with an open coach type interior of railway vehicles.

The effect of safety belts in railway vehicles is studied for instance in [10]. The authors employ both numerical models and physical tests with Hybrid III dummies to assess potential benefits of two-point seat belts. This study concludes that application of two-point belts increases passenger injuries in a crash situation in the majority of situations. In [4], the authors 
investigate the application of both two-point and three-point seat belts for the injury prevention of passengers in railway vehicles. They employ physical tests and numerical simulations with multi-body models representing anthropometric test devices (dummies). The authors conclude that application of two-point belts does not have a significant effect on the predicted injury of head and neck. Three-point belts, on the other hand, reduce the injury risk of a belted passenger. The effect of additional loading of the seats caused by seat belts is studied in [5]. In this study, physical tests and numerical simulations with dummies confirm overloading of seats causing failure of the seat frame.

The aim of this study is to contribute to the research of passenger's safety in railway vehicles. While the current studies employ anthropometric test devices, we choose a different model to represent a passenger. Virthuman is a human body model developed to represent real human body in various crash scenarios. Its application allows to evaluate potential benefit of seat belts in terms of passenger's injury risk with consideration of soft tissue deformation. Also, additional loading of seats caused by seat belts is evaluated in this study. We focus especially on the passenger vs table impact. Such impact has already been studied regarding workstation tables in [15]. However, we consider a different scenario with a passenger impacting folding table and the backseat. The paper is organized as follows. In Section 2 the computational models used in this study are presented. These involve the virtual human body model Virthuman, the interior of a railway vehicle and the model of seat belts. Section 3 describes the configurations of numerical simulations. We consider a passenger with no belt, the two-point belt and the three-point belt with both folded and unfolded tables. That is, we consider 6 configurations of numerical simulations in total. Section 4 analyzes all the results regarding kinematics and injury risk of the passenger as well as the loading of the seat during the scenario. Finally, conclusions are provided in Section 5.

\section{Computational models}

All the computational models presented in this study are developed in the Virtual Performance Solution (VPS) software, the Crash-PAM module, see [19]. It is a software designed for numerical analyses of fast dynamical processes such as impact scenarios using Finite Element (FE) models or Multi-Body Structures (MBS). It contains solver as well as tools for preprocessing and postprocessing of the simulation. In our case, explicit solver is used. Particular types of finite elements are specified for each particular part of the model. The theory of FE methods is provided, e.g., in [3], some examples of models in VPS software can be found in [18].

\subsection{Virtual human body model Virthuman}

As a representative of a passenger, the Virthuman model is used in the VPS software. It is based on multi-body structure with deformable segments. The skeleton of the model is formed of rigid bodies that are interconnected via kinematic joints to represent real joints of a human body. Moreover, additional joints are considered for particular bones to account for fractures. The surface of the model is formed of segments that are connected to the skeleton via nonlinear springs and dampers, see Fig. 1. Due to these deformable segments, deformation of soft tissues is considered in collision scenarios. The model has been validated in various test scenarios including individual body parts as well as whole body tests to ensure its biofidelity. That is, mechanical response of the model under various loading is in a good agreement with the mechanical response of a real human body, see, e.g., [20]. 

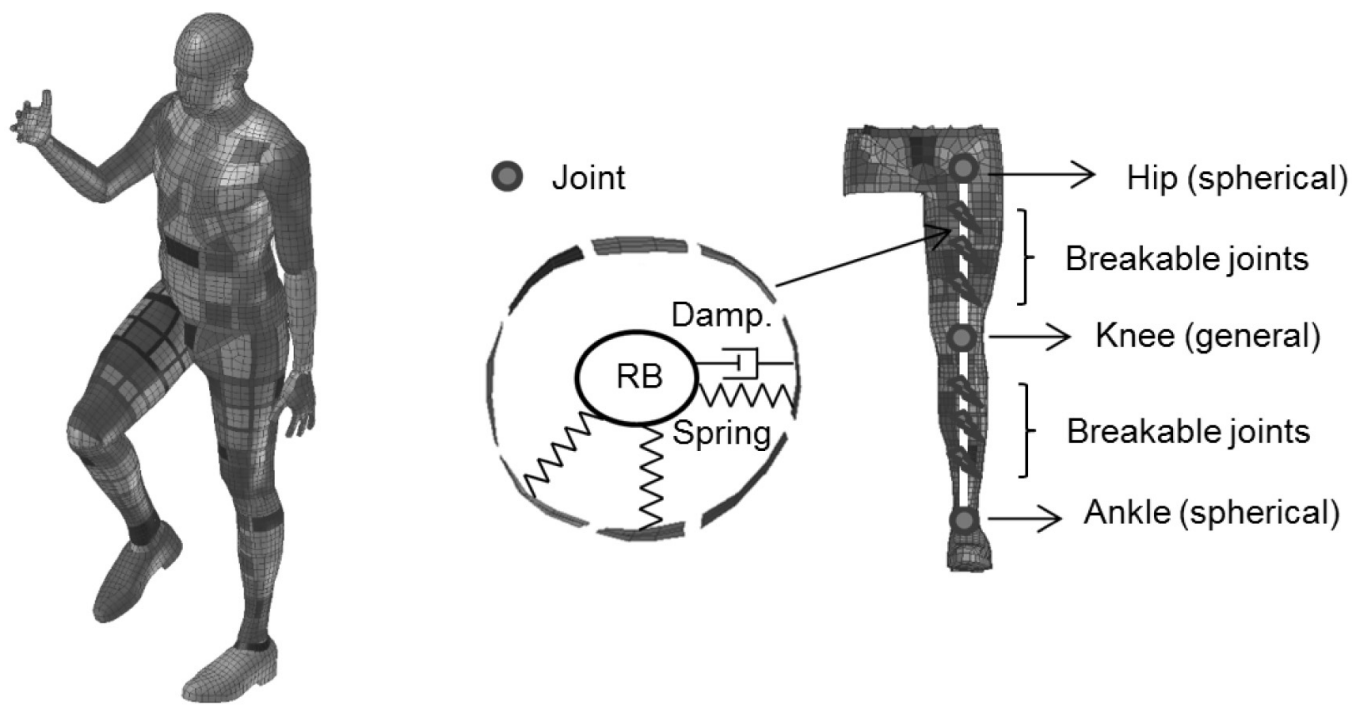

Fig. 1. Computational model Virthuman (left) and its segmentation (right)

The scaling algorithm has been developed for the model based on anthropometric dataset of more than 10 thousands volunteers of both Czech and Slovak population. Using this algorithm, referential model representing 50th percentile male can be scaled according to four input parameters. These are gender, age, height and weight. For this particular study, 25-year-old male of a 50th percentile is considered, that is, an average male with the height of $178 \mathrm{~cm}$ and the weight of $76 \mathrm{~kg}$. For details on the scaling of the model, see [11].

An important feature of the model is the embedded algorithm for the automatic evaluation of injury risk. During the simulation of a collision scenario, more than 700 curves representing forces, displacements, accelerations etc., of individual body parts are being recorded. Using these data, injury criteria are calculated. Exceeding of certain criterion is indicated with a colour in accordance with the EuroNCAP consumer rating [9]. Red colour represents "poor conditions", orange colour represents "marginal", yellow colour stands for "acceptable" and green for the "good conditions". The list of injury criteria currently evaluated in the Virthuman model is provided in Table 1 .

Table 1. Injury criteria evaluated in the Virthuman model

\begin{tabular}{|l|l|}
\hline Body part & Injury criteria \\
\hline Head & HIC36, HIC15 \\
\hline Neck & Upper neck My, tension, shear \\
\hline Thorax & Deflection (front, side), Viscous criterion (front, side) \\
\hline Abdomen & Compression force \\
\hline Pelvis & Pubic peak force \\
\hline Femur & Bending moment, Compression force \\
\hline Knee & Moments \\
\hline Tibia & Bending moment, Compression force \\
\hline
\end{tabular}

\subsection{Interior of a railway vehicle}

An open coach type interior is considered. Its model is developed in the VPS software. The passenger's seat is composed of the seat, the backrest and the headrest. Here, one particular seat is considered that is already being produced. Its parts are represented with deformable FE 


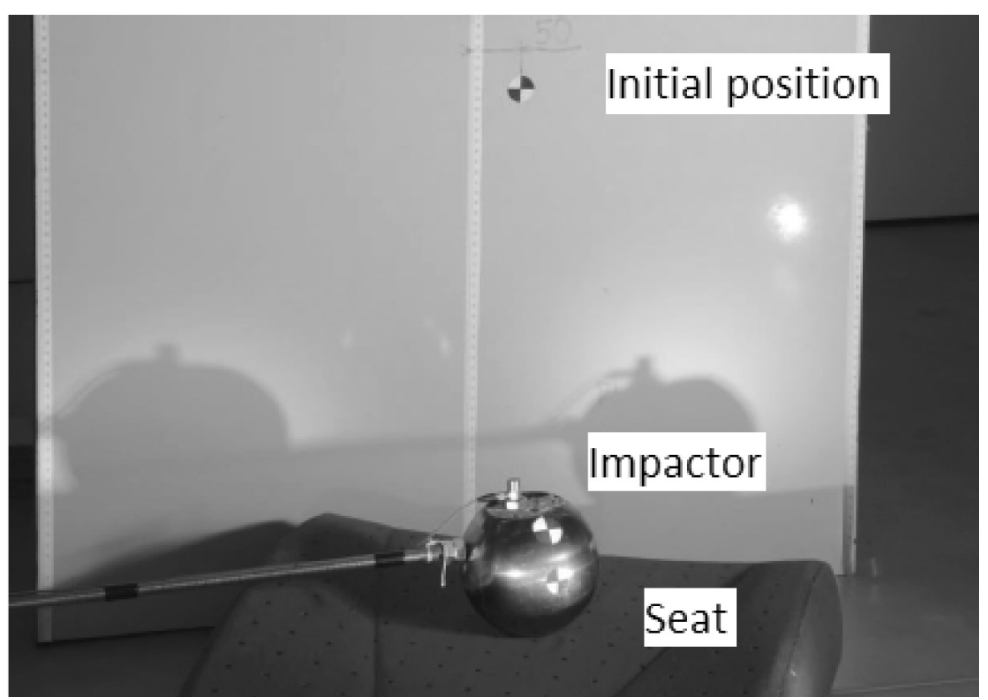

Fig. 2. Experimental setting [17]
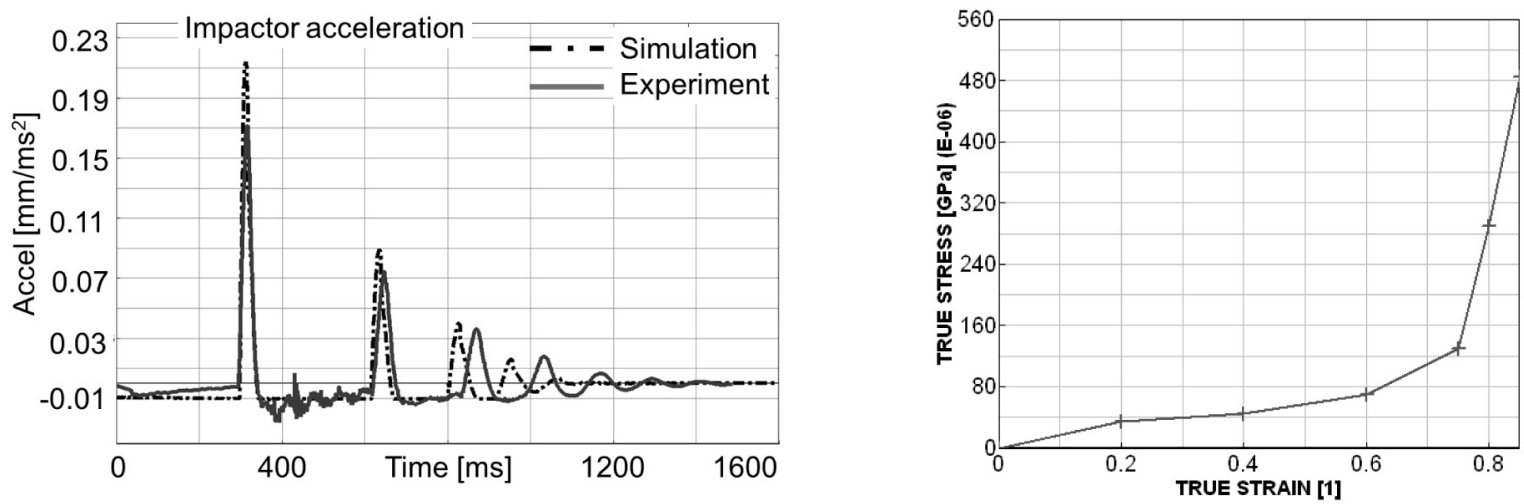

Fig. 3. Comparison of experimental data and model prediction in terms of impactor acceleration [17] (left). Dependency of true stress on the true strain of the foam material (right)

models. Total number of approximately 8500 hexahedral elements with the size of $20 \mathrm{~mm}$ per seat is considered. General nonlinear strainrate dependent foam with optional energy absorption is considered as a material model in the VPS software. This is to account for deformations of the seat upon the loading caused by the seated passenger. The material parameters of the model are determined on the basis of the experiment with a real foam that is described in [17], see Fig. 2. In this experiment, steel impactor is used to impact the foam in a drop test considering two different initial heights. Numerical simulations of the same tests are performed. Calibration of the material parameters of the model results in a good agreement with the experimental data in terms of acceleration of the impactor, see Fig. 3 (left). Resulting material characteristics, i.e., the dependency of the true stress on the true strain used in the model of the foam parts is depicted in Fig. 3 (right). The foam parts are used in the model of the passenger's seat only in order to have a proper seating of the passenger. In fact, the model of the passenger's seat is simplified in order to decrease the computational time.

Regarding the rear part of a seat in front of a passenger, an impact is expected during the accident scenario. Therefore, the model of the front seat is more detailed in comparison to passenger's seat to ensure accurate results of passenger impact. It is composed of closed steel profiles (shell with $2 \mathrm{~mm}$ thickness), cover (shell with $3 \mathrm{~mm}$ thickness) and backrest (shell with 
$5 \mathrm{~mm}$ thickness). For these materials, true stress-strain characteristics are used (steel DOCOL 1200, namely). For the definition of all shells, Belytschko-Tsay uniform reduced integration scheme is employed. Regarding the table, FE model of stoppers is used. It enables the table folding with the angle of $90^{\circ}$ representing stiffness of real stoppers. The response of a mechanism with gas piston is considered in the model via definitions of kinematic joints. The resulting model of a railway vehicle interior is shown in Fig. 4 (top). It is composed of two passengers seats with foam parts. In front of them, two seats with tables and realistic representations of structure are placed. In fact, the figure illustrates the configuration of the seated Virthuman model with the three-point belt and the folded table. The detail of the folded and unfolded table is illustrated in Fig. 4 (bottom).
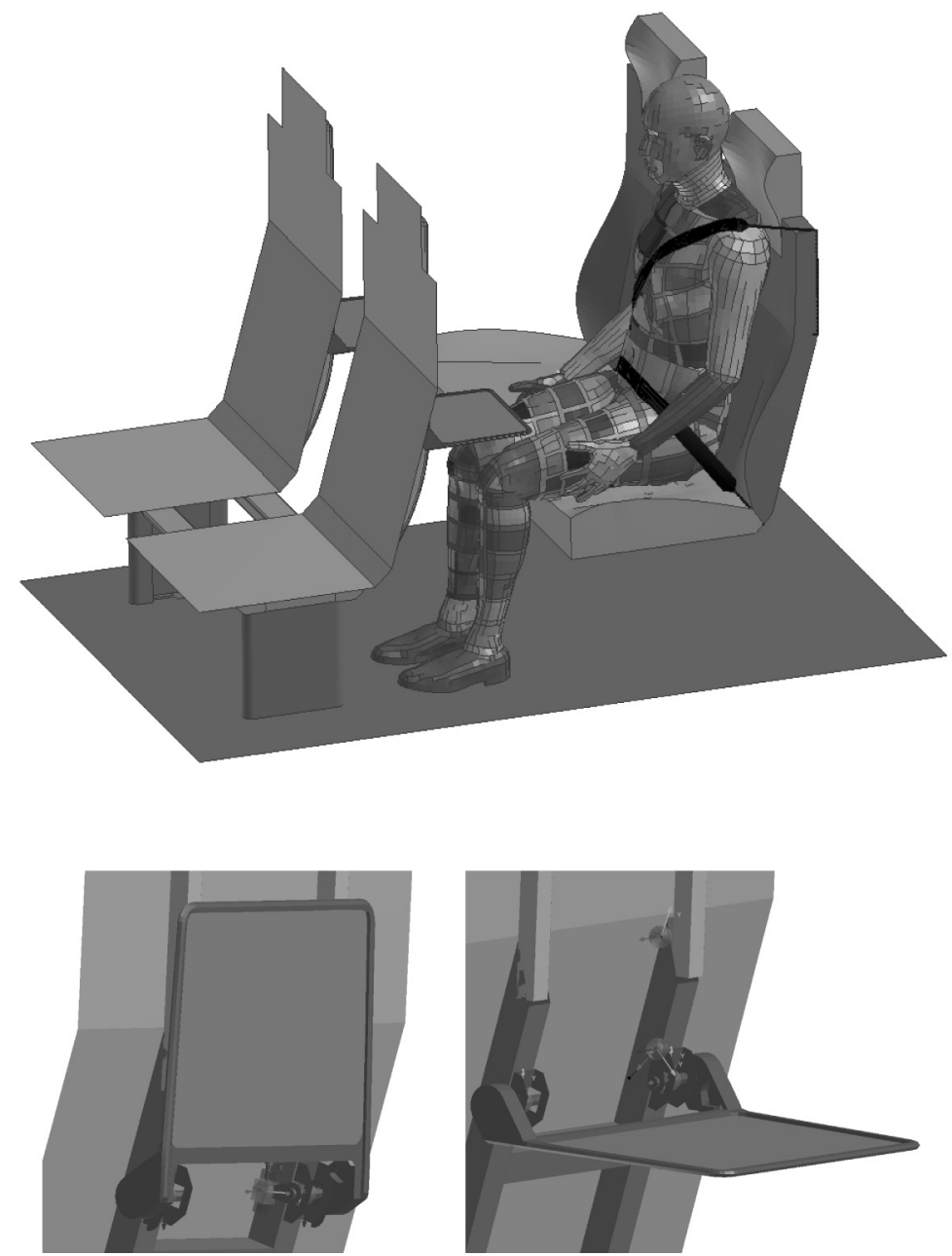

Fig. 4. Computational model of a railway vehicle interior (top). Detail of the unfolded and folded table in the model of the seat without the plastic cover (bottom)

\subsection{Safety belts}

In this study, two-point and three-point belt models are developed and embedded in the railway vehicle interior. Standard two-point belt that is common for passenger seats in buses is considered here. Three-point belt is also a standard one commonly used in cars. Their attachment to the seat model is illustrated in Fig. 5. Both belts are attached to the skeleton of the seat that is represented with the layer of shell elements and considered as a rigid body. Two-point belt is 

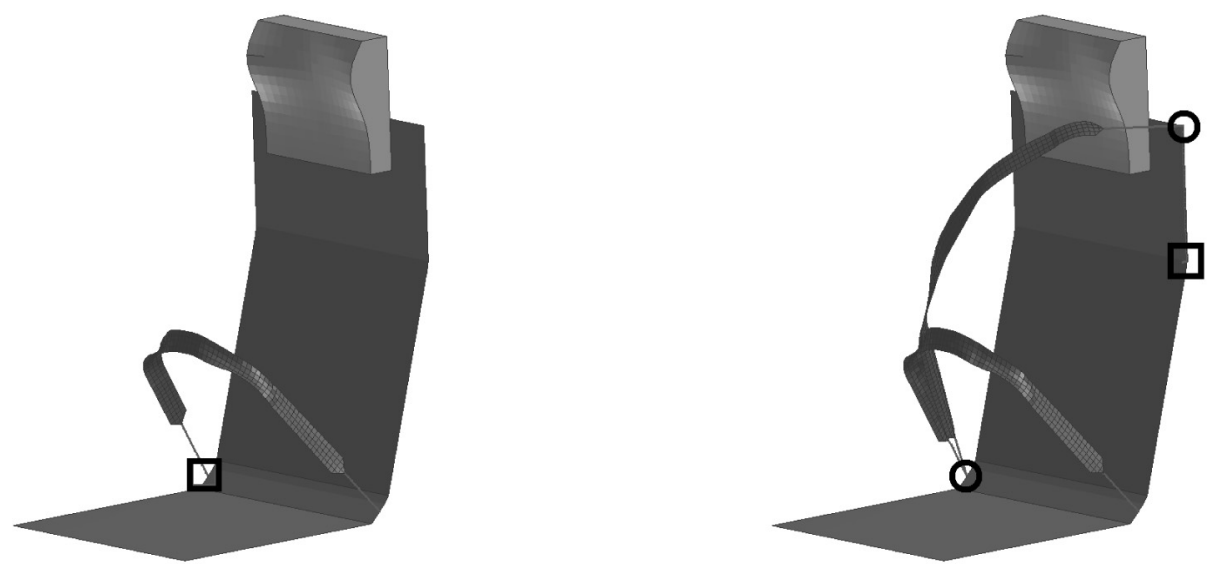

Fig. 5. Model of a two-point (left) and a three-point safety belt (right). The square marks the position of retractor, the circles mark the positions of sliprings. The geometry corresponds to a belt fastened by a 50th percentile male

anchored at the left part of the seat. At the right part, the retractor enables reeling of the belt with prescribed force. The retractor itself is fixed to the skeleton of the seat at its right part (marked in Fig. 5 with square). The three-point belt is anchored at the same position, i.e., at the left part of the skeleton. At the right side, the belt runs through the slipring attached to the skeleton (representation of a buckle). At the left side it runs through the slipring attached to the upper part of skeleton. Finally, the seat belt runs along the seat to the retractor. Anchorage of safety belts to the seat skeleton is considered with no additional constructions and enforcements of the skeleton. Among others, the aim of numerical simulations is to determine the loading of the seat and to determine whether such anchorage is sufficient.

The belts are represented with a layer of four-noded membrane elements. These have none bending stiffness and are suitable especially for modelling of fabrics. At the locations of sliprings, membrane elements are replaced with tension only bar elements. Here, friction is defined. Definition of a retractor force is used to simulate the seat belt fastening. Material parameters in the model are chosen to represent common seat belts in passenger vehicles.

\section{Configurations of numerical simulations}

In each configuration of a collision scenario, the human body model is seated at first. It is positioned in an upright sitting position in accordance with the GM/RT2100 regulation. The gravity only is applied for the duration of $100 \mathrm{~ms}$ to establish contact between the body and the seat. In configurations with the seat belts, the retractor force of $35 \mathrm{~N}$ is applied in order to fasten the seat belts and to establish contacts. The result of the simulation after $100 \mathrm{~ms}$ thus represents real seating of a passenger and fastening the seat belts.

For the collision scenario, an average 50th percentile male is considered (25-years old, $178 \mathrm{~cm}, 76 \mathrm{~kg}$ ) in a configuration with no belt, two-point belt and the three-point belt. Each of these cases is considered in a configuration with folded and unfolded table at the backrest in front of the passenger. It means, 6 configurations of numerical simulations are considered in total. The configuration with the three-point belt and the folded table is illustrated in Fig. 4.

After the first $100 \mathrm{~ms}$, locking of the retractor is prescribed. At this moment, acceleration pulse is prescribed for the whole interior in a frontal direction according to the GM/RT2100 regulation. In case of physical tests it is difficult to achieve precise acceleration curve. Therefore, 
the GM/RT2100 regulation prescribes a corridor for the acceleration pulse that corresponds to common frontal collision scenarios of a railway vehicle. The acceleration pulse has to fit the prescribed corridor. In our numerical simulations, we choose the central curve of the corridor as the acceleration pulse, see Fig. 6. The whole simulation time is $400 \mathrm{~ms}$. All the simulations are prepared, processed and analyzed using the VPS software.

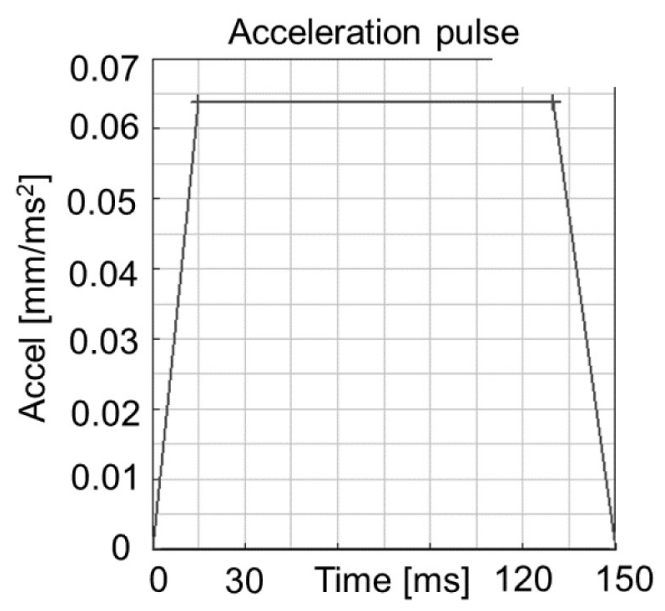

Fig. 6. Acceleration pulse according to the GM/RT2100 regulation

\section{Results}

\subsection{Passenger kinematics}

Kinematics of the passenger with no safety belt is illustrated in Fig. 7. Here, both configurations with unfolded (left) and folded table (right) are provided at certain time levels, namely at $95 \mathrm{~ms}$, $150 \mathrm{~ms}$ and $185 \mathrm{~ms}$. The time levels are considered with respect to the start of loading with acceleration pulse.

We discuss here also the possible injury caused by impact with the backrest. However, the injury analysis with respect to EuroNCAP and GM/RT2100 regulations is provided in Section 4.2 and summarized in Tables 2 and 3.

For both configurations (folded and unfolded table), the kinematics is in fact similar. At the approximate time of $95 \mathrm{~ms}$, knees impact the backrest possibly causing the injury of both femurs. At the approximate time of $150 \mathrm{~ms}$, the head hits the headrest causing possible neck injury. In the case of the unfolded table, slightly higher head injury risk is also indicated. At the approximate time of $185 \mathrm{~ms}$, there is a significant difference in these two configurations. In the case with the unfolded table, the thorax and abdomen hit the backrest just in the location of the table. Since the table is made of steel, the impact causes higher injury risk of these two body parts. In the case of the folded table, no impact of the thorax and abdomen with the backseat occurs.

Kinematics of the passenger with two-point belt is illustrated in Fig. 8 (left). Since both table configurations provide similar results, the configuration with unfolded table is illustrated only. From top to bottom, time levels of $115 \mathrm{~ms}, 150 \mathrm{~ms}$ and $175 \mathrm{~ms}$ are provided. In contrast to the configuration with no belt, no primary impact of lower limbs and the backrest occur. Hence, no injury risk of the lower limbs is indicated. The body of the passenger is fixed by the two-point belt at the abdominal area. However, the torso and lower limbs are thrown against 

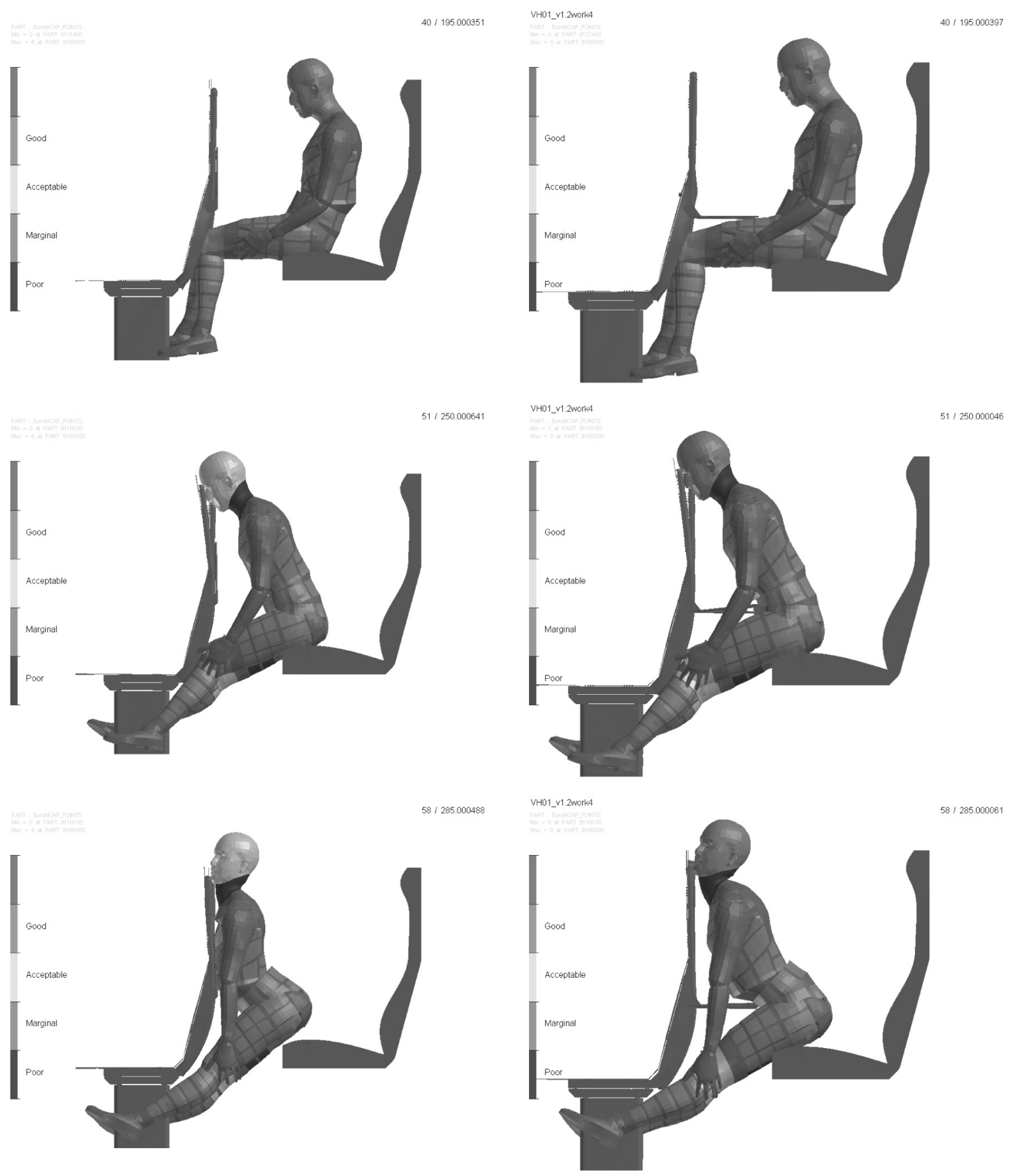

Fig. 7. Kinematics of the passenger during the collision scenario with no belt. Unfolded (left) and folded table (right). Time levels from top to bottom at $95 \mathrm{~ms}, 150 \mathrm{~ms}$ and $185 \mathrm{~ms}$

the backrest, as it can bee seen at the time level of $115 \mathrm{~ms}$. The abdomen is compressed by the belt causing higher injury risk of this body part. At the approximate time of $150 \mathrm{~ms}$, the head hits the backrest. In the configuration with unfolded table the head hits right the table causing serious risk of head injury (poor conditions). In the configuration with folded table the head hits the plastic part of the backrest which does not increase the head injury risk. During the ongoing collision scenario the torso is being bent until the contact of the thorax and lower limbs, see the time level of $175 \mathrm{~ms}$ in Fig. 8 (left). This causes increased loading of neck and consequently its higher injury risk (poor conditions in both configurations). 

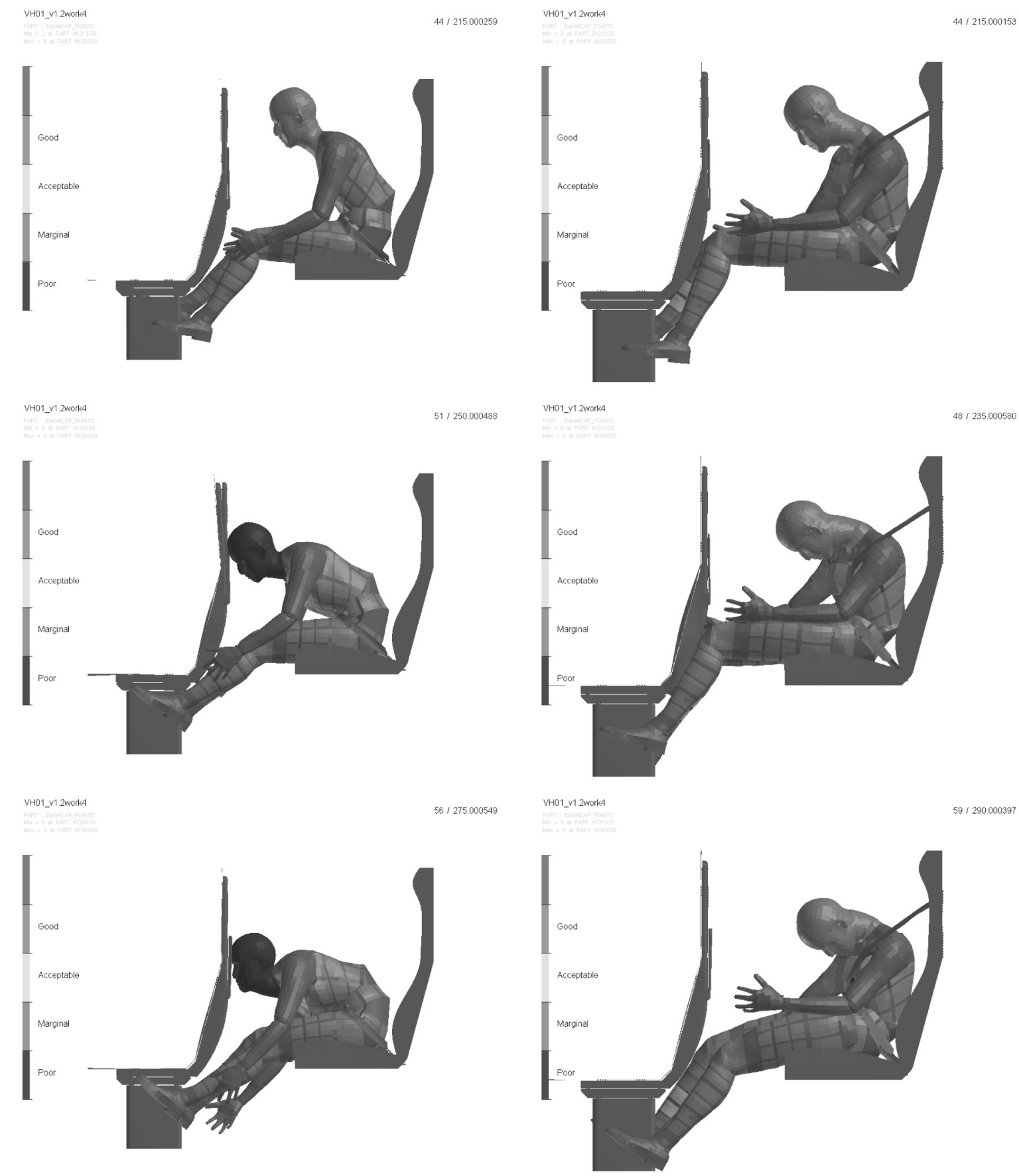

Fig. 8. Kinematics of the passenger during the collision scenario with two-point belt (left) and the threepoint belt (right). Configuration with unfolded table is illustrated. Time levels for the two-point belt (left) from top to bottom at $115 \mathrm{~ms}, 150 \mathrm{~ms}$ and $175 \mathrm{~ms}$. Time levels for the three-point belt (right) from top to bottom at $115 \mathrm{~ms}, 135 \mathrm{~ms}$ and $190 \mathrm{~ms}$

Kinematics of the passenger with three-point belt is illustrated in Fig. 8 (right). In this case, both table configurations provide nearly the same results. Hence, the configuration with unfolded table is illustrated only. From top to bottom, time levels of $115 \mathrm{~ms}, 135 \mathrm{~ms}$ and $190 \mathrm{~ms}$ are provided. In this case, whole upper part of the body is fixed with the belt, hence it does not impact the backrest in front of the passenger. At the approximate time of $115 \mathrm{~ms}$, the upper part of the body is compressed by the belt causing the slightly increased injury risk (abdomen, 
thorax). Also, right tibia hits the rear part of the seat causing higher injury risk of this body part. At the approximate time of $135 \mathrm{~ms}$, the lower limbs are in contact with the backrest, however, the injury risk is not increased. At the time of $190 \mathrm{~ms}$, the body is getting back to the seat due to the forces acting in the belt. Clearly, the three-point belt minimizes the impact of the passenger with the other parts of interior. It results in the lowest injury risk predicted (among all three cases).

\subsection{Injury analysis}

In this section, the injury risk of passenger in each configuration of collision scenario is assessed. Each body part is evaluated with respect to the EuroNCAP regulation (Table 2) and the GM/RT2100 regulation (Table 3). In Table 2, individual boxes are denoted with a code number with respect to the exceeded limits. That is, we represent "poor conditions" with (4), "marginal" with (3), "acceptable" with (2) and "good conditions" with (1). The number coding is used instead of colours for the purposes of this particular paper printed in grayscale. In the case of exceeding certain injury criteria, the value is provided as well. Similarly to EuroNCAP, the GM/RT2100 regulation also prescribes injury criteria and corresponding limits. However, its application is in railway vehicles collision scenarios and hence the criteria and limits may differ from those used in automotive industry. Therefore, there are no code numbers in Table 3. Here, chosen injury criteria are evaluated. Their exceeding is provided with a particular value. If the criterion does not exceed the limit value, corresponding box is marked with a " $\checkmark$ " sign.

Table 2. Evaluation of injury criteria according to EuroNCAP regulation. We represent "poor conditions" with (4), "marginal" with (3), "acceptable" with (2) and "good conditions" with (1)

\begin{tabular}{|c|c|c|c|c|c|c|}
\hline & \multicolumn{3}{|c|}{ Unfolded table } & \multicolumn{3}{|c|}{ Folded table } \\
\hline Body part & No belt & 2-point belt & 3-point belt & No belt & 2-point belt & 3-point belt \\
\hline Head & (2) & (4) & (1) & $(1)$ & (1) & (1) \\
\hline HIC36 & 736 & 1593 & 58 & 605 & 607 & 44 \\
\hline Neck & (4) & $(4)$ & (1) & (4) & (4) & (1) \\
\hline Moment [Nm] & 303 & -138 & & 304 & -107 & \\
\hline Thorax & (3) & (3) & (4) & (1) & (3) & (4) \\
\hline Deflection/VC & $0.67 \mathrm{~m} / \mathrm{s}$ & $40 \mathrm{~mm}$ & $63 \mathrm{~mm}$ & & $34 \mathrm{~mm}$ & $59 \mathrm{~mm}$ \\
\hline Abdomen & (3) & (3) & $(2)$ & (1) & (3) & $(2)$ \\
\hline Force $[\mathrm{kN}]$ & 2.14 & 1.77 & 1.5 & & 1.76 & 1.6 \\
\hline Pelvis & $(1)$ & $(1)$ & (1) & (1) & $(1)$ & (1) \\
\hline Femur left & (3) & (1) & (1) & (3) & (1) & (1) \\
\hline Force $[\mathrm{kN}]$ & 8.6 & & & 8.4 & & \\
\hline Femur right & (4) & (1) & (1) & (4) & (1) & (1) \\
\hline Force $[\mathrm{kN}]$ & 9.3 & & & 9.2 & & \\
\hline $\begin{array}{l}\text { Knee left } \\
\text { Moment }[\mathrm{Nm}]\end{array}$ & (1) & (1) & (1) & $\begin{array}{l}(2) \\
101\end{array}$ & (1) & (1) \\
\hline Knee right & (2) & (1) & (1) & (2) & (1) & (1) \\
\hline Moment [Nm] & 109 & & & 107 & & \\
\hline Tibia left & (1) & (1) & (1) & (1) & (1) & (1) \\
\hline Tibia right & (1) & (1) & (2) & (1) & (1) & (2) \\
\hline Moment [Nm] & & & 228 & & & 228 \\
\hline
\end{tabular}


Table 3. Evaluation of injury criteria according to GM/RT2100 regulation

\begin{tabular}{|c|c|c|c|c|c|c|}
\hline & \multicolumn{3}{|c|}{ Unfolded table } & \multicolumn{3}{|c|}{ Folded table } \\
\hline Body part & No belt & $2 p$ & $3 p$ & No belt & $2 p$ & $3 p$ \\
\hline $\begin{array}{l}\text { Head } \\
\text { HIC15 }<500\end{array}$ & 502 & 1593 & $\checkmark$ & $\checkmark$ & 607 & $\checkmark$ \\
\hline $\begin{array}{l}\text { Neck } \\
-4 \mathrm{kN}<\text { Force }<4.17 \mathrm{kN} \\
-135 \mathrm{Nm}<\text { Moment }<310 \mathrm{Nm}\end{array}$ & $-4.3 \mathrm{kN}$ & $-138 \mathrm{Nm}$ & $\checkmark$ & $\checkmark$ & $-8.9 \mathrm{kN}$ & $\checkmark$ \\
\hline $\begin{array}{l}\text { Thorax } \\
\text { Deflection }<63 \mathrm{~mm}\end{array}$ & $\checkmark$ & $\checkmark$ & $63 \mathrm{~mm}$ & $\checkmark$ & $\checkmark$ & $\checkmark$ \\
\hline $\begin{array}{l}\text { Abdomen } \\
\text { Deflection }<40 \mathrm{~mm}\end{array}$ & $\checkmark$ & $\checkmark$ & $\checkmark$ & $\checkmark$ & $\checkmark$ & $\checkmark$ \\
\hline $\begin{array}{l}\text { Femur left } \\
\text { Force }<5.7 \mathrm{kN}\end{array}$ & $8.6 \mathrm{kN}$ & $\checkmark$ & $\checkmark$ & $8.4 \mathrm{kN}$ & $\checkmark$ & $\checkmark$ \\
\hline $\begin{array}{l}\text { Femur right } \\
\text { Force }<5.7 \mathrm{kN}\end{array}$ & $9.3 \mathrm{kN}$ & $\checkmark$ & $\checkmark$ & $9.2 \mathrm{kN}$ & $\checkmark$ & $\checkmark$ \\
\hline $\begin{array}{l}\text { Tibia left } \\
\text { Force }<8 \mathrm{kN}\end{array}$ & $9 \mathrm{kN}$ & $\checkmark$ & $\checkmark$ & $9 \mathrm{kN}$ & $\checkmark$ & $\checkmark$ \\
\hline $\begin{array}{l}\text { Tibia right } \\
\text { Force }<8 \mathrm{kN}\end{array}$ & $8.8 \mathrm{kN}$ & $\checkmark$ & $\checkmark$ & $9.5 \mathrm{kN}$ & $\checkmark$ & $\checkmark$ \\
\hline
\end{tabular}

Let us discuss each configuration with respect to results in Tables 2 and 3 . In the case of a passenger with no belt, injury of lower limbs is predicted for both folded and unfolded tables. This is the result of knee impact with the steel profiles in the backrest. Limits for both left and right femurs are exceeded. The EuroNCAP regulation predicts also a minor injury risk of knees. According to GM/RT2100 regulation, limits for axial force in tibiae are exceeded. In configuration with unfolded table, the head impact with the headrest causes slight exceeding of limit values for HIC36 and HIC15, respectively. Both regulations predict also neck injury in this case. The kinematics is slightly different in the case with the folded table. The head impact does not cause exceeding of the limits. EuroNCAP regulation, however, predicts neck injury. Moreover, EuroNCAP regulation predicts risk of thorax and abdomen injury in configuration with unfolded table due to its impact with these body parts.

In the case of two-point belt, the body is fixed in the abdominal region. No knee impact with the backrest occurs and hence no injury risk of lower limbs is predicted. According to both regulations, there is a critical impact of head with unfolded table leading to high injury risk of both head and neck. In the case of the folded table, the head impacts with plastic part of the backseat and hence no serious head injury risk is predicted. Still, neck injury risk is increased also in the case of the folded table. EuroNCAP regulation also predicts injury risk of abdomen and thorax caused by the belt and by leaning the thorax on lower limbs.

In the case of the three-point belt, whole upper part of the body is fixed. Therefore, some minor impacts of lower limbs with the backrest are observed only. According to EuroNCAP regulation, such impacts lead to slighly higher injury risk in right tibia. No injury risk is predicted for both head and neck. According to EuroNCAP regulation, limits are exceeded for thorax and abdomen due to the belt itself. In case of GM/RT2100 regulation, the exact limit value, i.e., $63 \mathrm{~mm}$, is reached for the thorax deflection in unfolded table configuration. 


\subsection{Seat loading}

In this section we focus on the loading of the seat itself during the collision scenario. The contact forces between the passenger and the backrest as well as forces acting in seat belts are evaluated. The reason is to assess the need of additional reinforcement of seats in the case when seat belts are in use. We can assume that current interior of a railway vehicle is designed to bear the loading caused by 50th percentile male during the collision scenario as defined by the GM/RT2100 regulation. That is, if the load transmitted via seat belts is lower, no additional reinforcement is needed.

At first, we focus on the configuration with no seat belts, namely the one with unfolded table. Two major impacts occur between passenger and the seat in front of the passenger. The first one is the knee impact at approximate time of $95 \mathrm{~ms}$ resulting in the contact force exceeding $10 \mathrm{kN}$. The second one is the head impact at approximate time of $150 \mathrm{~ms}$ leading to the contact force exceeding $5 \mathrm{kN}$.

In configuration with the two-point seat belt, the axial forces at each side of the belt are evaluated. The results in case with unfolded table are provided in Fig. 9 (left). The results are nearly the same also for the case with folded table. We observe maximal loads of approximately $5.4 \mathrm{kN}$ and $3.6 \mathrm{kN}$ at each side, respectively. The sum of these forces is provided as well. In total, the force acting on the seat does not exceed $10 \mathrm{kN}$. That is, application of a two-point belt does not require additional reinforcement of the seat.

In three-point belt, the axial forces near each of the fixation points are evaluated. The results are provided in Fig. 9 (right). Clearly, the sum of individual forces does not exceed $10 \mathrm{kN}$ which represent the overall loading of the seat. Thus again, application of a three-point belt does not require additional reinforcement of the seat.
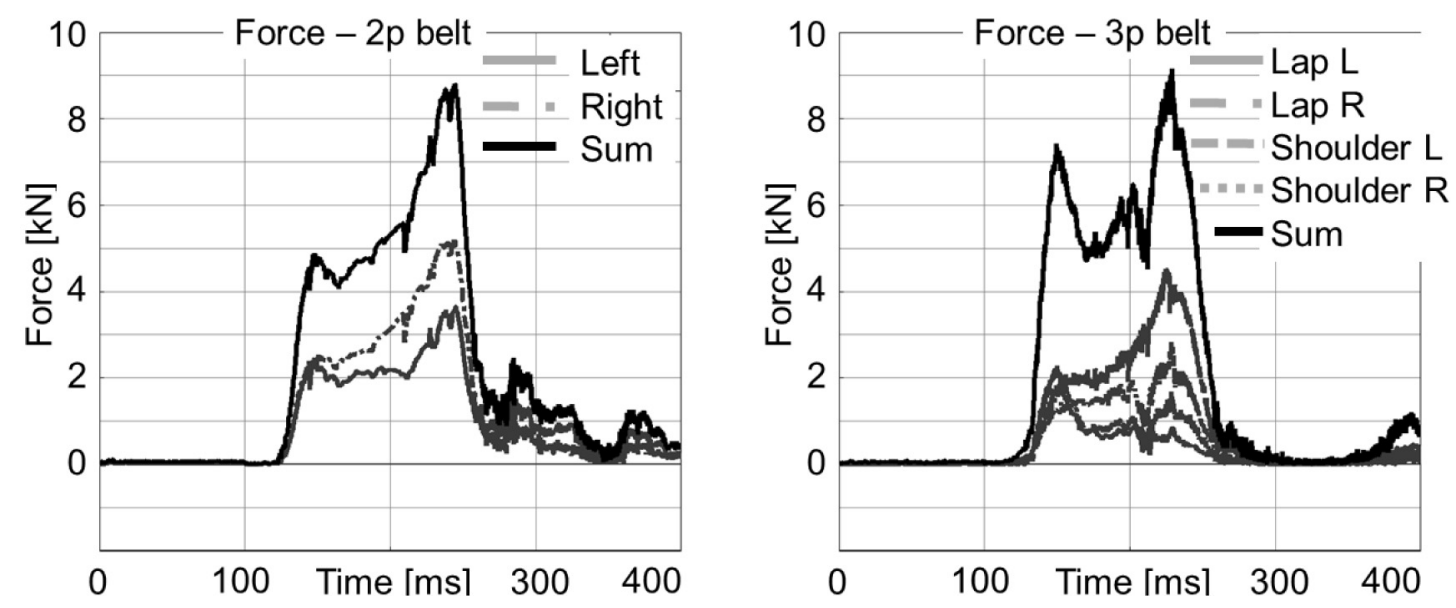

Fig. 9. Time history of belt forces. Two-point belt with corresponding forces at each side and their sum (left). Three-point belt with corresponding forces near each of its fixation points and their sum (right)

\section{Conclusions}

In this work, numerical simulations of a collision scenario of a railway vehicle were performed as defined by the GM/RT2100 regulation. Computational model of a railway vehicle interior has been developed and the acceleration pulse has been prescribed. The passenger (50th percentile male) was represented with the virtual human body model Virthuman $(25$-years old, $178 \mathrm{~cm}$, 
$76 \mathrm{~kg}$ ). The configurations with no constraints, two-point belt and the three-point belt were considered. Each configuration was tested with both folded and unfolded table. This resulted in 6 numerical simulations in total. With respect to the results obtained, following conclusions can be formulated:

- With no seat belt, high injury risk of lower limbs is predicted as a result of the impact with steel profiles. The head impact with the headrest leads to increased head injury risk. Increased injury risk of these body parts is predicted also in [4].

- Injury risk of lower limbs is completely reduced in the case of the two-point belt. This is confirmed for the 50th percentile male also in [4]. However, this type of belt significantly increases the head injury risk in the case of the unfolded table. Also, significant neck injury risk is predicted in the case of the two-point belt for both the folded and unfolded tables.

- In this particular frontal impact, application of a two-point seat belt does not lead to an increase of passenger's safety. This finding is in agreement with studies regarding buses [1] as well as other studies regarding railway vehicles $[4,10]$.

- Application of a three-point belt minimizes injury risk of both head and neck. The only possible injury might occur as a result of interaction of the belt with the thorax and abdomen. With respect to the passenger's safety, the three-point belt is the most suitable one. Minimization of injury risks with the three-point belt is also confirmed in [4].

- In numerical simulations, fixation of the belts with the current construction of seats is considered and the acting forces are evaluated. These forces do not exceed the forces acting during impact with passenger in configuration with no belt. Therefore, application of seat belts should not require additional reinforcements of seats. On the contrary, the study [5] concludes that application of seat belts may lead to the seat overloading and failure of the seat frame. This might be caused by different seat types used in the studies. In our case, the seat with a high strength steel frame is considered complying the GM/RT2100 regulation.

- The simulations suggest that in the case of mounting seat belts in a railway vehicle interior of an open coach type, three-point belts should be preferred to two-point belts. In the case of a frontal collission scenario as defined by the GM/RT2100 regulation, the overall injury risk of a passenger is lower for the three-point belt when compared to the two-point belt.

In the present study, one particular collision scenario and one particular interior type was considered. Also, the passenger was represented with a 50th percentile male only to meet the test conditions as prescribed by the GM/RT2100 regulation. However, this might be considered as a limitation of the current study. To generalize our conclusions, extension beyond test conditions of the GM/RT2100 regulation might be favourable. That means to consider also different collision scenarios, various railway vehicle interior types and also various anthropometries to represent passengers. These might be suggestions for a further progress. 


\section{Acknowledgements}

This work has been supported by the project TRIO FV20441 "Research and development of safe tram face" provided by the Ministry of Industry and Trade of Czech Republic.

\section{References}

[1] Albertsson, P., Falkmer, T., Is there a pattern in European bus coach incidents? A literature analysis with special focus on injury causation and injury mechanisms, Accident Analysis and Prevention 37 (2005) 225-233. https://doi.org/10.1016/j.aap.2004.03.006

[2] Björnstig, U., Albertsson, P., Björnstig, J., Bylund, P.-O., Falkmer, T., Petzäll, J., Injury events among bus and coach occupants, IATSS Research 29 (2005) 79-87. https://doi.org/10.1016/S0386-1112(14)60121-7

[3] Bonet, J., Wood, R. D., Nonlinear continuum mechanics for a finite element analysis, Cambridge University Press, Cambridge, 1997.

[4] Caputo, F., Lamanna, G., Fidanza, F., Multibody investigation on the passive safety performances of seats in railway vehicles, Proceedings of the 10th Biennial on Engineering System Design and Analysis ASME/ESDA, Istanbul, Turkey, 2010, pp. 195-202. https://doi.org/10.1115/ESDA2010-24766

[5] Caputo, F., Lamanna, G., Soprano, A., On the evaluation of the overloads coming from the use of seat-belts on a passenger railway seat, International Journal of Mechanics and Materials in Design 8 (2012) 335-348. https://doi.org/10.1007/s10999-012-9199-1

[6] ČSN, Railway applications - Crashworthiness requirements for railway vehicle bodies, ČSN EN 15227, 2011. (in Czech)

[7] Drobný, V., Panenka, J., Vašíček, M., Tichý, Z., Improved passenger's crash safety in coach by frontal collision, Proceedings of the Computational Mechanics conference, Nečtiny, 2010, pp. 1-2.

[8] ECE Regulation No. 66, Uniform technical prescriptions concerning the approval of large passenger vehicles with regard to the strength of their superstructure, 2009.

[9] EuroNCAP, Assessment protocol - Adult occupant protection, version 6.0, July 2013.

[10] Freeman, J., Matthews, P., Improving the design of seats and tables, and evaluating restraints to minimise passenger injuries - Review of two-point passenger restraints, Rail Safety and Standards Board Ltd., London, 2005.

[11] Hyncik, L., Cechova, H., Kovar, L., Blaha, P., On scaling virtual human models, SAE Technical Paper 2013-01-0074, 2013. https://doi.org/10.4271/2013-01-0074

[12] Le Corre, D., Crash pulse definitions of the SAFEINTERIORS project, Proceedings of the 8th International Symposium on PASSIVE SAFETY of Rail Vehicles, 32, 2011, pp. 65.

[13] Nakai, K., Simulation of passenger behavior on board a commuter train in the event of a level crossing accident, Proceedings of the 9th International Symposium on PASSIVE SAFETY of Rail Vehicles, 43, 2013, pp. 53-62.

[14] RSSB, Requirements for rail vehicle structures, GM/RT2100, 2012.

[15] Severson, K., Research and development of a safety standard for workstation tables in the United States, Proceedings of the 9th International Symposium on PASSIVE SAFETY of Rail Vehicles, 43, 2013, pp. 107-121.

[16] Snape, P., Developing a crashworthy rail seating system using finite element analysis, Proceedings of the 9th International Symposium on PASSIVE SAFETY of Rail Vehicles, 43, 2013, pp. 123-132.

[17] Špirk, S., Methodological problems of passive safety research and deformation resistance design of rail vehicles, Ph.D. thesis, University of West Bohemia, Pilsen, 2015. (in Czech) 
[18] Tutorial and material calibration examples for PAM-CRASH, PAM-FORM and PAM-OPT. http://www2.ifb.uni-stuttgart.de/fem/TutorialsAndMaterialCalibration.htm

[19] Virtual Performance Solution, software.

http://www.esi-group.com/software-solutions/virtual-performance/virtual-performance-solution

[20] Vychytil, J., Manas, J., Cechova, H., Spirk, S., Hyncik, L., Kovar, L., Scalable multi-purpose virtual human model for future safety assessment, SAE Technical Paper 2014-01-0534, 2014. https://doi.org/10.4271/2014-01-0534 